\title{
A.В. БОРЦОВ
}

\section{АППРОКСИМАЦИЯ ВОЛЬТАМПЕРНОЙ ХАРАКТЕРИСТИКИ ВЕНТИЛЬНОЙ СОЛНЕЧНОЙ БАТАРЕИ}

\begin{abstract}
Вольтамперная характеристика является основной функциональной характеристикой солнечной батареи. Она позволяет определить оптимальный, с точки зрения передачи максимальной мощности в нагрузку, режим работы солнечной батареи. Теоретически вольтамперная характеристика вентильного фотоэлемента описывается системой нелинейных дифференциальных уравнений 5-го порядка с соответствующими граничными условиями. Система уравнений представляет собой баланс электронов и дырок внутри вентильного фотоэлемента. Распределение напряженности электрического поля описывается уравнением Пуассона. Аналитическое решение данной системы возможно в двух случаях - для “тонкого” p-n перехода и для $p$ - $n$ перехода, внутри которого можно пренебречь процессами генерации и рекомбинации носителей заряда. Аналитическое решение в данном случае представляет собой уравнение Эберса-Молла, которое связывает между собой ток, напряжение в нагрузке и параметры фотоэлемента - фотоэдс, фототок, ток насыщения, тепловой потенциал. В общем случае система уравнений решается численными методами. В литературе численные результаты представляют уравнением Эберса-Молла, в которое введен коэффициент $a=1-5$. В работе предложен экспериментально-расчетный метод определения параметров фотоэлемента и коэффициента $a$. Некоторые параметры фотоэлемента можно измерить в процессе снятия вольтамперной характеристики - фотоэдс в режиме холостого хода и фототок в режиме короткого замыкания. Для определения параметра $а$ и тока насыщения по экспериментальным данным использован метод наименьших квадратов. Предложенный метод аппроксимации вольтамперной характеристики применен к нескольким кремниевым поликристаллическим солнечным батареям.

Ключевые слова: солнечная батарея, вольтамперная характеристика, $p$ - $n$ переход, фотоэдс, ток насыщения, тепловой потенциал, “тонкий” $p$ - $n$ переход, “широкий” $p$ - $n$ переход, уравнение Эберса-Молла
\end{abstract}

\section{О.В. БОРЦОВ}

\section{АПРОКСИМАЦІЯ ВОЛЬТАМПЕРНОЇ ХАРАКТЕРИСТИКИ ВЕНТИЛЬНОЇ СОНЯЧНОЇ БАТАРЕЇ}

Вольтамперна характеристика $є$ основною функціональною характеристикою сонячної батареї. Вона дозволяє визначити оптимальний, з точки зору передачі максимальної потужності в навантаження, режим роботи сонячної батареї. Теоретично вольтамперна характеристика вентильного фотоелемента описується системою нелінійних диференціальних рівнянь 5-го порядку з відповідними граничними умовами. Система рівнянь являє собою баланс електронів і дірок всередині вентильного фотоелемента. Розподіл напруженості електричного поля описується рівнянням Пуассона. Аналітичне рішення даної системи можливо в двох випадках - для "тонкого" $p$ - $n$ переходу і для $p$ - $n$ переходу, всередині якого можна знехтувати процесами генерації і рекомбінації носіїв заряду. Аналітичне рішення в даному випадку є рівняння ЕберсаМолла, яке пов'язує між собою струм, напругу в навантаженні і параметри фотоелемента - фотоерс, фотострум, струм насичення, тепловий потенціал. У загальному випадку система рівнянь вирішується чисельними методами. У літературі чисельні результати представлено рівнянням Еберса-Молла, в яке введено коефіцієнт $a=1-5$. В роботі запропоновано експериментально-розрахунковий метод визначення параметрів фотоелемента і коефіцієнта $a$. Деякі параметри фотоелемента можливо виміряти в процесі зняття вольтамперної характеристики - фотоерс в режимі холостого ходу і фотострум в режимі короткого замикання. Для визначення параметра $a$ і струму насичення за експериментальними даними використано метод найменших квадратів. Запропонований метод апроксимації вольтамперної характеристики застосовано до кількох кремнієвих полікристалічних сонячних батарей.

Ключові слова: сонячна батарея, вольтамперна характеристика, $p$ - $n$ перехід, фотоерс, струм насичення, тепловий потенціал, "тонкий" $p$ $n$ перехід, "широкий" p- $n$ перехід, рівняння Еберса-Молла

\section{A.V. BORTSOV}

\section{APPROXIMATION OF CURRENT-VOLTAGE CHARACTERISTIC OF VENTILED SOLAR BATTERY}

The current-voltage characteristic is the main functional characteristic of the solar battery. It allows you to determine the optimal, in terms of transferring maximum power to the load, the mode of operation of the solar battery. Theoretically, the current-voltage characteristic of a valve photocell is described by a system of non-linear differential equations of the 5th order with the corresponding boundary conditions. The system of equations is the balance of electrons and holes inside the valve photocell. The distribution of the electric field strength is described by the Poisson equation. An analytical solution of this system is possible in two cases - for the "thin" $p-n$ junction and for the p-n junction, inside which the processes of generation and recombination of charge carriers can be neglected. The analytical solution in this case is the Ebers-Mall equation, which interconnects the current, voltage in the load and the parameters of the photocell - photoemf, photocurrent, saturation current, thermal potential. In the general case, the system of equations is solved by numerical methods. In the literature, numerical results are represented by the Ebers-Mall equation, in which the coefficient $\mathrm{a}=1-5$ is introduced. An experimental-calculation method for determining the parameters of the photocell and coefficient a is proposed. Some parameters of the photocell can be measured in the process of taking the current-voltage characteristic - photoemf in idle mode and photocurrent in short circuit mode. The least squares method was used to determine the parameter a and the saturation current from the experimental data. The proposed method for approximating the current-voltage characteristic is applied to several silicon polycrystalline solar batteries.

Keywords: solar battery, current-voltage characteristic, p-n junction, photoemf, saturation current, thermal potential, "thin" p-n junction, "wide" p-n junction, Ebers-Mall equation.

Введение. Основная функциональная характеристика любого источника энергии - вольтамперная характеристика (BAX) или нагрузочная характеристика, которая показывает зависимость тока от напряжения на зажимах источника. При этом линейный источник энергии можно представить как реальный источник напряжения с внутренним сопротивлением. Величину напряжения и внутреннего сопротивления можно рассчитать или измерить. ВАХ такого источника - прямая линия. Для расчета или измерения параметров линейного источника используют режим холостого хода и короткого замыкания [1]. Солнечная батарея

Вісник Національного технічного університету «ХПІ». Серія: Проблеми 
представляет собой нелинейный источник. Теоретически ВАХ солнечной батареи можно рассчитать, решив систему нелинейных дифференциальных уравнений. Аналитическое решение этой системы возможно в случае “тонкого" $p-n$ - перехода или $p-n$ - перехода, внутри которого можно пренебречь процессами генерации и рекомбинации носителей заряда [2]. В остальных случаях система уравнений решается численными методами

Цель работы - экспериментальное исследование и аппроксимация ВАХ вентильной солнечной батареи.

Постановка задачи. ВАХ кремниевой солнечной батареи можно аналитически аппроксимировать уравнением Эберса - Молла [2, 3]:

$$
U(I)=\frac{a k T}{e} \ln \left(\frac{I_{f}-I}{I_{s}}+1\right),
$$

где $k=1,38 \cdot 10^{-23}$, Дж/К - постоянная Больцмана; $e=$ $1,6 \cdot 10^{-19}$, Кл - заряд электрона; $I_{f}-$ фототок; $I_{s}-$ ток насыщения; $T$ - абсолютная температура; $a$ - коэффициент, учитывающий “ширину” $p$ - $n$ - перехода.

Коэффициент $a$ предлагается выбирать из диапазона $a=1-5$, причем $a=1$ - для “тонкого" $p$ - $n$ - перехода, и $a=2-5$ - для “широкого” $p-n-$ перехода. Для дальнейшего анализа приведем уравнение (1) к безразмерному виду. Введем базисные величины:

$I_{\sigma}=I_{f}-$ базисный ток (фототок); $U_{\sigma}=\frac{k T}{e}=U_{T}-$ базисное напряжение (тепловой потенциал). Тогда уравнение ВАХ в безразмерном виде запишется как:

$$
u(i)=a \ln \left(\frac{1-i}{i_{s}}+1\right),
$$

где $u(i)=\frac{U(I)}{U_{\sigma}} ; i=\frac{I}{I_{б}} ; i_{s}=\frac{I_{s}}{I_{б}}$.

В уравнении (2) два неизвестных параметра - коэффициент $a$ и относительный ток насыщения $i_{s}$. В режиме холостого хода $(i=0)$ из уравнения (2) получим

$$
i_{s}=1 /\left[\exp \left(\frac{\varepsilon_{f}}{a}\right)-1\right],
$$

где $\varepsilon_{f}=\frac{U(0)}{U_{\sigma}}=\frac{E_{f}}{U_{T}}-$ относительная фотоэдс солнечной батареи. Таким образом, уравнение (2) можно записать в виде:

$$
u(i)=a \ln \left[(1-i) e^{\frac{\varepsilon_{f}}{a}}+i\right],
$$

то есть параметр $а$ является критерием подобия ВАХ солнечной батареи [4]. Фототок $I_{f}$, фотоэдс $E_{f}$ и тепловой потенциал $U_{T}$ определяются в процессе снятия ВАХ солнечной батареи.

Описание стенда для снятия ВАХ и результаты аппроксимации. Схема стенда для снятия ВАХ солнечной батареи представлена на рис. 1.

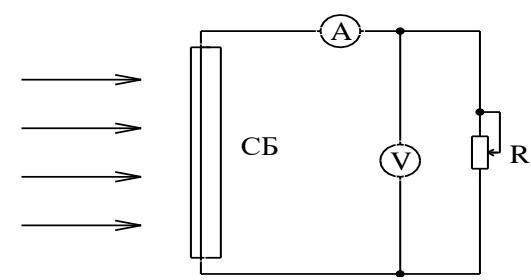

Рис. 1. Схема стенда для экспериментальных исследований: СБ - солнечная батарея; $A$ - амперметр; $V$ - вольтметр; $R$ - нагрузка.

Объектом экспериментальных исследований являлась кремниевая солнечная батарея площадью

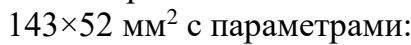

- ток короткого замыкания (фототок) - 6,2 мА;

- напряжение холостого хода (фотоэдс) - 7,69 В;

- схема соединения солнечных элементов - 2 параллельные цепочки по 16 последовательно соединенных элементов;

- размер одного элемента $-25 \times 8$ мм². $^{2}$

Солнечная батарея при комнатной температуре $\left(T \approx 301^{\circ} \mathrm{K}\right)$ облучалась световым потоком, создаваемым энергосберегающей лампой GLOBAL мощностью 75 Вт. Измерения токов, напряжений и температуры проводились цифровыми мультиметрами M890G, DG890B+. В качестве нагрузки использован многооборотный проволочный резистор СП3-37А сопротивлением 51 кОм. Результаты измерений $U=U(I)$ приведены в таблице 1 и на рис. 2.

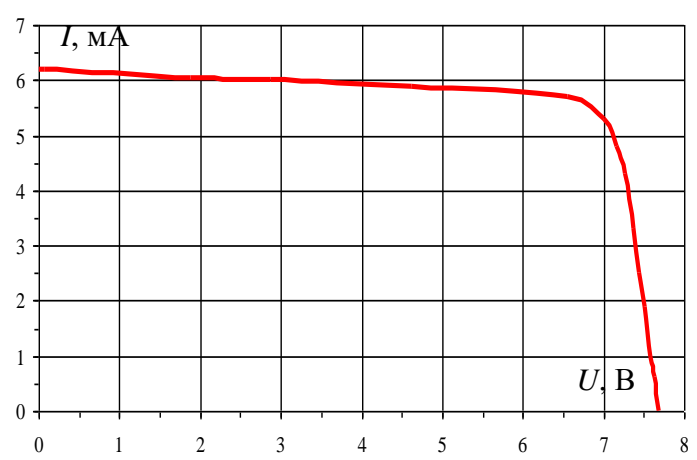

Рис. 2. Экспериментальная ВАХ солнечной батареи.

Таблица 1 - Результаты измерений ВАХ солнечной батареи.

\begin{tabular}{|c|c|c|c|c|c|c|c|c|c|c|c|c|c|}
\hline$I$, мA & 0,00 & 0,62 & 1,00 & 1,90 & 2,50 & 3,50 & 4,30 & 5,00 & 5,48 & 5,85 & 6,03 & 6,07 & 6,20 \\
\hline$U, \mathrm{~B}$ & 7,69 & 7,63 & 7,59 & 7,52 & 7,45 & 7,37 & 7,27 & 7,17 & 7,00 & 6,56 & 4,62 & 2,88 & 0,00 \\
\hline
\end{tabular}

Экспериментальная ВАХ имеет безразмерный вид $u_{k}=u_{k}\left(i_{k}\right)$, где $k=1,2 \ldots n$. Число точек измерения $\mathrm{BAX} n=13$ (табл. 1). Для определения параметра $a$ по результатам измерений используем метод “наименьших квадратов” [5]. Составим “невязку” ВАХ

$$
S=\sum_{\mathrm{k}=1}^{\mathrm{n}}\left\{a \ln \left[\left(1-i_{k}\right) e^{\frac{\varepsilon_{f}}{a}}+i_{k}\right]-u_{k}\right\}^{2} .
$$

Минимизируя “невязку” $d S / d a=0$, получим трансцендентное уравнение для определения параметра $a$, которое решаем методом итераций [5]. В результате 
получим:

$$
\begin{gathered}
a^{(m+1)}=\frac{1}{\sum_{k=1}^{(n-1)} \ln ^{2} f\left(i_{k}\right)} \sum_{k=1}^{(n-1)}\left\{u_{k} \ln f\left(i_{k}\right)+\right. \\
\left.+\frac{\varepsilon_{f}\left(1-i_{k}\right)}{f\left(i_{k}\right)} e^{\frac{\varepsilon_{f}}{a^{(m)}}}\left[\frac{u_{k}}{a^{(m)}}+\ln f\left(i_{k}\right)\right]\right\} ; \\
\left.f\left(i_{k}\right)=\left(1-i_{k}\right) \exp { }^{\frac{\varepsilon_{f}}{a^{(m)}}}\right)+i_{k} ; \\
a^{(0)}=\sum_{k=1}^{(n-1)}\left(u_{k}-\varepsilon_{f}\right) \ln \left(1-e^{\varepsilon_{f}^{(m)}}-1\right] ; \sum_{k=1}^{(n-1)} \ln ^{2}\left(1-i_{k}\right) ; \\
\left|a^{(m+1)}-a^{(m)}\right| \leq \varepsilon,
\end{gathered}
$$

$m=0,1,2 . .-$ число итераций, которое задается погрешностью $\varepsilon \leq 0,05$. После нескольких итераций $(m \leq 5-6)$ получим $a=12,53 ; I_{s}=3,33 \cdot 10^{-7} \mathrm{~A}$. Сравнение расчетных и экспериментальных ВАХ показывает хорошее совпадение (рис. 3). Но значение параметра $a$

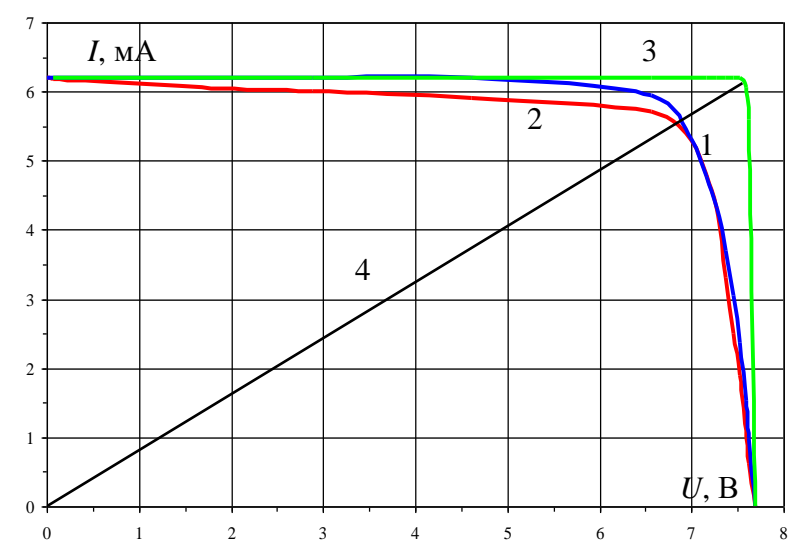

Рис. 3. Сравнение расчетной (1) и экспериментальной (2) вольтамперной характеристик солнечной батареи; (3) вольтамперная характеристика солнечной батареи с “тонким" $p-n$ переходом; (4) - прямая максимальной мощности далеко от диапазона $a=1-5$. Построим ВАХ одного фотоэлемента - для этого значения тока батареи разделим на 2, а значения напряжения на 16. Расчет по выражениям (6 - 10) для одного фотоэлемента дают $a=1,903 ; I_{s}=3,71 \cdot 10^{-7}$ А. Следовательно, для одного фотоэлемента значение параметра $a$ уже соответствует диапазону $a=1-5$.

Выводы. 1. Предложен экспериментальный метод определения параметров ВАХ кремниевой солнечной батареи. Применение этого метода для конкретного типа солнечной батареи показало хорошее совпадение расчетной и экспериментальной ВАХ.

2. Установлено, что приводимый в литературе диапазон параметра $a=1-5$ справедлив только для одного фотоэлемента.

\section{Список литературы}

1. Нейман Л.Р., Демирчян К.С. Теоретические основы электротехники: В 2-х т. Учебник для вузов. Том 1. Л.: Энергоиздат. Ленингр. отд-ние, 1981. - 536 с.

2. Васильев А.М., Ландсман А.П. Полупроводниковые фотопреобразователи. М.: Изд-во «Советское радио». 1971. - 248 с.

3. Хоровиц П., Хилл У. Искусство схемотехники: В 2-х томах. Пер. с англ. М.: Мир. 1983. - Т. 1. -598 с.

4. Веников В.А. Теория подобия и моделирования (применительно к задачам электроэнергетики). Учеб. пособие для вузов. Изд. 2е, доп. и перераб. М.: «Высшая школа», 1976. - 479 с.

5. В.П. Демидович, И.А. Марон, Э.З. Шувалова. Численные методы анализа. Государственное издательство физико-математической литературы. М.: 1962. - 367 с.

\section{References (transliterated)}

1. Neyman L.R., Demirchyan K.S. Teoreticheskiye osnovy elektrotekhniki: V 2-kh t. Uchebnik dlya vuzov. Tom 1. L.: Energoizdat. Leningr. otd-niye, 1981. - $536 \mathrm{~s}$.

2. Vasil'yev A.M., Landsman A.P. Poluprovodnikovyye fotopreobrazovateli. M.: Izd-vo «Sovetskoye radio». 1971. -248 s.

3. .Khorovits P., Khill U. Iskusstvo skhemotekhniki: V 2-kh tomakh. Per. s angl. M.: Mir. 1983. - T.1. 598 s.

4. Venikov V.A. Teoriya podobiya i modelirovaniya (primenitel'no $\mathrm{k}$ zadacham elektroenergetiki). Ucheb. posobiye dlya vuzov. Izd. 2-ye, dop. i pererab. M.: «Vysshaya shkola», 1976. - 479 s.

5. V.P. Demidovich, I.A. Maron, E.Z. Shuvalova. Chislennyye metody analiza. Gosudarstvennoye izdatel'stvo fiziko-matematicheskoy literatury. M.: $1962 .-367 \mathrm{~s}$.

\section{Відомості про авторів / Сведения про авторов / About the authors}

Борцов Олександр Васильович (Борцов Александр Васильевич, Bortsov Alexander Vasilievich) - кандидат технічних наук, доцент, Національний технічний університет «Харківський політехнічний інститут», доцент кафедри інженерної електрофізики; м. Харків, Україна; email: avbortsov@gmail.com. 